\title{
White Supremacy, Anti-Black Racism, and Mathematics Education: Local and Global Perspectives
}

\author{
Luz Valoyes-Chávez, Danny B. Martin, Joi Spencer and Paola Valero
}

Understanding the forms wherein racism operates in mathematics teaching and learning practices requires taking into account the local meanings of race and the composing features of racialized social systems. The mechanisms through which racism is reproduced within school mathematics across racialized social systems differ subtly, but manifest themselves in strikingly similar ways. So, while the meanings of race are malleable and locally situated, its production of privilege, power, exemption, and disenfranchisement is stable. Likewise, the still widespread view that mathematics and mathematics education are non-political practices has allowed little interrogation of how racialized social practices permeate mathematics education. The minimization of attention to racism in international mathematics education research is perplexing, particularly in contexts characterized by long histories of anti-Blackness and colonialism and in other contexts that are facing increased levels of xenophobia, anti-immigration, and contestations of national identity.

The Discussion Group was an opportunity to create awareness on these issues and to push for analyses of race and racism in local, global, and international contexts. We aimed at facilitating discussion among colleagues around the world on experiences of racisms and racialization of mathematics education. We also intended to find and imagine different ways of collaborative work that move the field forward and have an impact on research, practice, and policy. Around 30 participants mainly from the United States, England, Brazil, South Africa, Portugal, and Germany took part in the discussions. An initial recalling of experiences that could be seen as instantiations of racism in relation to math education was a starting point for recognizing the overt as well as the subtle ways in which racism is instantiated and becomes present. In some contexts, being the "black body" is associated with incapacity for mathematical thinking. In other contexts, the color of skin as a marker intersects with belonging to

\footnotetext{
L. Valoyes-Chávez $(\bowtie)$

Universidad Santiago de Cali, Cali, Colombia

e-mail: luz.valoyes00@usc.edu.co

(C) The Author(s) 2017

G. Kaiser (ed.), Proceedings of the 13th International Congress on Mathematical

Education, ICME-13 Monographs, DOI 10.1007/978-3-319-62597-3_109
} 
other categories such as "minority," "immigrant," or "indigenous." An important realization was that participants who would consider themselves concerned with diversity and equity were surprised by not only how racism can operate inside well-intentioned and progressive views of mathematical participation but also how well-intentioned progressivism in mathematics education can allow racism to maintain its role in shaping the larger social order. The aim of questioning the conflation of notions of race with "black bodies" was achieved as participants' contexts allowed them to evidence the local meanings and practices of racism.

Group leaders presented some key contemporary notions of race and racialization in connection to the notion of white supremacy. The reflection on how assumed characteristics of universality and abstraction of mathematics embedded in notions of school mathematics are part of the narratives of mathematics as the invention of White cultures were challenged. A significant concluding point was the importance of questioning the White epistemologies on which widespread ideas of mathematics education build. Such exploration could become an area of international collaboration for practitioners and scholars interested in broadening participation in mathematics education practices for many students who have been opted out through the operation of racialized epistemologies of mathematics education.

Open Access Except where otherwise noted, this chapter is licensed under a Creative Commons Attribution 4.0 International License. To view a copy of this license, visit http://creativecommons. org/licenses/by/4.0/.

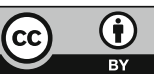

